\title{
Biogenic Magnetite in Humans and New Magnetic Resonance Hazard Questions
}

\author{
O. Strbak ${ }^{1}$, P. Kopcansky ${ }^{2}$, I. Frollo ${ }^{1}$ \\ ${ }^{1}$ Institute of Measurement Science, Department of Imaging Methods, Slovak Academy of Sciences, \\ Dubravska cesta 9,84104 Bratislava, Slovakia, oliver.strbak@savba.sk \\ ${ }^{2}$ Institute of Experimental Physics, Department of Theoretical Physics, Slovak Academy of Sciences, \\ Watsonova 47, 04001 Kosice, Slovakia
}

\begin{abstract}
The widespread use of magnetic resonance (MR) techniques in clinical practice, and recent discovery of biogenic ferrimagnetic substances in human tissue, open new questions regarding health hazards and MR. Current studies are restricted just to the induction of Faraday currents and consequent thermal effects, or 'inoffensive' interaction with static magnetic field. We outlined that magnetic energies associated with interaction of ferrimagnetic particles and MR magnetic fields can be dangerous for sensitive tissues like the human brain is. To simulate the interaction mechanism we use our 'Cube' model approach, which allows more realistic calculation of the particle's magnetic moments. Biogenic magnetite nanoparticles face during MR examination three principal fields: (i) main $B_{0}$ field, (ii) gradient field, and (iii) $B_{1}$ field. Interaction energy of biogenic magnetite nanoparticle with static magnetic field $B_{0}$ exceeds the covalent bond energy 5 times for particles from $4 \mathrm{~nm}$ up to $150 \mathrm{~nm}$. Translation energy in gradient field exceeds biochemical bond energy for particles bigger than $50 \mathrm{~nm}$. Biochemical bond disruption and particle release to the tissue environment, in the presence of all MR fields, are the most critical points of this interaction. And together with relaxation processes after application of RF pulses, they make biogenic magnetite nanoparticles a potential MR health hazard issue.
\end{abstract}

Keywords: Biogenic magnetite, ‘Cube’ model, magnetic resonance, health hazard

\section{INTRODUCTION}

$\mathrm{T}$ HE MAGNETITE is one of the oldest magnetic materials known to mankind (around $2000 \mathrm{BC}$ ). It is ferrimagnetic and crystallizes in the inverse cubic spinel structure.

Large magnetite crystals, in order to minimize their internal energy, have developed multiple magnetic domains. On the other hand, in smaller crystals there is only one magnetic domain, so the whole crystal has a large magnetic moment resistant to a change in direction. Crystals that fall below single domain size range become superparamagnetic - susceptible to random changes in magnetic orientation due to thermal vibrations [1].

The process of magnetite biomineralization in living systems was first discovered in marine molluscs of the class Polyplacophora [2]. Since then, the biogenic magnetite was found in several biological species, including mammals [3]. Magnetite biomineralization is a genetically-controlled biochemical process, through which organisms make perfect ferrimagnetic crystals. However, the magnetosome formation process is still not well understood. This process evolved about two billion years ago in the magnetotactic bacteria, and presumably was incorporated in the genome of higher organisms, including humans [4]. Magnetite crystals produced by living systems have unique features that distinguish them from geologically produced crystals [5]. These involve (i) single domain size, (ii) chemical purity, (iii) crystallographic perfection, (iv) participation in chain structure, (v) unusual morphology and (vi) [111] elongation. These properties serve to maximize the net magnetic moment which affects the migratory and homing abilities of animal species in Earth's magnetic field. The magnetite- based magnetoreception assumes that magnetite nanoparticles are connected to nervous structures, and that the torque response exerted by the geomagnetic field on the particles is responsible for mechanical stresses on the surrounding cellular environment. Subsequently, the mechanical stress is transformed into nervous signals through the activation of membrane mechanoreceptors [6]. In 1992, Kirschvink et al. discovered the presence of biogenic magnetite in the human brain tissue [7]. Further magnetic measurements on samples of human tissue confirmed presence of trace quantities of stable (at room temperature) single domain magnetite particles in the brain [8], [9], [10] and other organs [11]. Although, several ideas about the biological function of magnetite in humans have been proposed, the real purpose is still not known. Several studies suggested that levels of biogenic magnetite in human brain tissue may be elevated in subjects with Alzheimer disease [12], [13], [14] or associated with aging [15]. It was proposed, that hypointensive artefacts found in $\mathrm{T}_{2}$ weighted images of human brain could correlate with presence of magnetite clusters [16]. It gives an opportunity for noninvasive detection of early stages of neurodegenerative processes, but still does not answer the question about the reason of biologically produced ferrimagnetic material in humans. On the other hand, presence of biogenic ferrimagnetic nanoparticles in such sensitive tissue raises new questions in MR safety. Current MR hazard issues are just restricted to induction of Faraday currents and consequent thermal effects. However, the magnetic properties of naturally occurring magnetite particles make them perfect candidates for biophysical response in external magnetic fields. Tissue with magnetite nanoparticles is 
exposed to strong static magnetic fields (100000 times higher than Earth's magnetic field), magnetic gradients and series of RF pulses during MR examination. This raises the question, how significant is interaction between particles embedded in living neural tissue and artificial magnetic sources for the safety of patients. We introduce preliminary ideas.

\section{SUBJECT \& METHODS}

Magnetite nanoparticles in external magnetic fields are generally approximated to a sphere with radius $\approx 10^{-7} \mathrm{~m}$ and magnetic moment $\approx 2 \times 10^{-15} \mathrm{Am}^{2}$ [17]. However, it is not a very realistic approximation and in our simulations it can lead to incorrect conclusions. To determine more realistic particle magnetic moment, we use our 'Cube' model method with two approaches: (i) cell unit (CU), and (ii) bulk [18].

In ' $C U$ ' approach, the magnetic moment of particle is derived from cell unit (CU) shape and size of the particle. Magnetite $\mathrm{CU}$ is made from 8 formula units (FU) and belongs to isometric - hexoctahedral crystal system (space group $\mathrm{Fd} 3 \mathrm{~m}$ ) with cell dimensions $a_{C U}=0.83958 \mathrm{~nm}$ and volume $V_{C U}=5.9182 \times 10^{-28} \mathrm{~m}$. The magnetic moment for the particle was calculated as follows:

$$
\vec{\mu}_{m a g}=N_{C U} \vec{\mu}_{C U}=8 N_{C U} \vec{\mu}_{F U}
$$

where $\mathrm{N}_{\mathrm{CU}}$ is the number of $\mathrm{CU}$ in particle

$$
N_{C U}=V_{\text {mag }} / V_{C U}
$$

and $\vec{\mu}_{F U}$ for magnetite was calculated by Huang [19]:

$$
\vec{\mu}_{F U}=4.33 \mu_{B} \approx 4 \times 10^{-23} \mathrm{Am}^{2}
$$

where $\mu_{B}$ is the Bohr magneton.

In 'Bulk' approach, the magnetic moment is derived from saturation magnetization constant of bulk magnetite $\left(\mathrm{M}_{\text {sat(Bulk })} \approx 90 \mathrm{Am}^{-2} \mathrm{~kg}^{-1}\right)$.

$$
\vec{\mu}_{\text {mag }(B u l k)}=M_{\text {sat }(B u l k)} m_{\text {mag }}
$$

where

$$
m_{m a g}=\frac{V_{m a g}}{V_{C U}} m_{C U}
$$

is the mass of the one magnetite nanoparticle. $\mathrm{V}_{\mathrm{mag}}$ is the particle volume ( $\left.V_{m a g}=a^{3}\right)$. Mass of the one 'cell unit' is calculated from molecular weight $\mathrm{M}_{\mathrm{r}}$ and atomic mass constant $\mathrm{m}_{\mathrm{u}}$ :

$$
m_{C U}=8 \mathrm{Mr}\left(\mathrm{Fe}_{3} \mathrm{O}_{4}\right) m_{u}
$$

We determined nanoparticles magnetization $\vec{M}_{\text {mag }}$ (which is size and temperature dependent) in magnetic field $\vec{B}$ applying the Langevin function:

$$
\vec{M}_{\text {mag }}=M_{s a t}[\operatorname{coth}(x)-1 / x]
$$

where $M_{s a t}$ is the saturation magnetization of particles and $x=\vec{\mu}_{\text {mag }} \vec{B} / k_{B} T$. The values of saturation magnetization $\mathrm{M}_{\text {sat }}$ for magnetite nanoparticles were determined experimentally (for $a=4 ; 11.5 ; 47.7 ; 150 \mathrm{~nm} \rightarrow \mathrm{M}_{\text {sat }}$ $=31.8 ; 60.1 ; 65.4 ; 75.6 \mathrm{Am}^{2} \mathrm{~kg}^{-1}$ at $T=300 \mathrm{~K} \mathrm{[20])}$.

For spherical particles, the magnetic moment was calculated in the same way as for cube particles in ' $\mathrm{CU}$ ' approach, with diameter equal to cube size ' $a$ '.

Biogenic magnetite nanoparticles are characteristics for its single magnetic domain. Single domain magnetic nanoparticles can interact with strong external magnetic fields in two ways: translation and rotation [21]. The maximum translation force occurs in the area where the static magnetic field gradient is maximal [22], and the maximum torque occurs in the area where the magnetic field is uniform and maximal [23].

Translation motion is caused by magnetic gradients $\vec{B}_{\text {grad }}$, acting on particle with magnetic force $\vec{F}_{m a g}$ :

$$
\vec{F}_{\text {mag }}=\left(\vec{\mu}_{\text {mag }} \cdot \vec{\nabla}\right) \vec{B}_{\text {grad }}
$$

Rotation is caused by interaction of particle magnetic moment $\vec{\mu}_{\text {mag }}$, with main magnetic field $\vec{B}_{0}$, which is $10^{5}$ bigger than the geomagnetic field. Torque $\vec{\tau}_{\text {mag }}$ :

$$
\vec{\tau}_{\text {mag }}=\vec{\mu}_{m a g} \times \vec{B}_{0}
$$

Torque acting on the particle changes during rotation and the work done by field in rotating particle from instantaneous position through an infinitesimally small angle $\mathrm{d} \theta$ is:

$$
d W_{\text {mag }}=\vec{\mu}_{\text {mag }} \vec{B} \sin \theta d \theta
$$

Viscosity of surrounding environment is acting against translation and rotation motion of magnetic nanoparticles, , with viscosity force $\vec{F}_{\text {visc }}$ [24]:

$$
\vec{F}_{v i s c}=3 \pi \eta d \vec{v}
$$

where $\eta$ - medium viscosity (for brain $\eta=3.5 \operatorname{Pa~s}[25]$ ), $d-$ particle diameter, $\vec{v}$ - particle velocity. 
The velocity of particle $\vec{v}_{m a g}$ in gradient field is such given by:

$$
\vec{v}_{\text {mag }}=\frac{\vec{\mu}_{\text {mag }}}{3 \pi \eta d} \frac{\partial B_{\text {grad }}}{\partial x}
$$

In the presence of rotational magnetic field $\vec{B}_{r o t}$, the angular velocity of particle $\vec{\omega}_{m a g}$ is given by:

$$
\vec{\omega}_{\text {mag }}=\frac{\vec{\mu}_{m a g} \times \vec{B}_{r o t}}{3 \pi \eta d(a / 2)}
$$

In standard clinical scanners, magnetic field gradients are produced by switchable EM coils. They are used to encode the spatial position of the signal and are quickly switched in a very short time. A typical modern clinical scanner is able to generate $40 \mathrm{mTm}^{-1}$ at a switching rate of up to $200 \mathrm{Tm}^{-1} \mathrm{~s}$ ${ }^{1}$, and the gradient can be switched from zero to maximum in $\approx 200 \mu$ s [26]. The main magnetic field $\vec{B}_{0}$ is in clinical scanners $1.5 \mathrm{~T}$ or $3 \mathrm{~T}$, respectively. However, $7 \mathrm{~T}$ wholebody tomography is used for research.

Biogenic magnetite nanoparticle inside human tissue faces three extreme fields during MR examination:

strong static magnetic field $\vec{B}_{0}$, (b) artificial magnetic gradient $\vec{B}_{\text {grad }}$ and (c) powerful RF pulses, the so called $\vec{B}_{1}$ field. Currently, the main attention is given to RF pulses, which are associated with SAR (specific absorption rate) calculations [27]. Biogenic ferrimagnetic material and its interaction with static and gradient magnetic field are usually neglected. We are focusing on energies associated with such interaction, in comparison with biological energies. Bond energies associated with biological molecules are shown in Table 1:

\begin{tabular}{|l|c|c|}
\hline Bond & Energy [kJ/mol] & Energy x10 \\
\hline Ionic & $\sim 700$ & 11.6 \\
\hline Covalent III & $800-1000$ & $13.3-16.6$ \\
\hline Covalent II & $500-700$ & $8.3-16.6$ \\
\hline Covalent I & $300-500$ & $4.98-8.3$ \\
\hline Dipole interact. & $40-400$ & $0.664-6.64$ \\
\hline Hydrogen & $10-40$ & $0.166-0.664$ \\
\hline
\end{tabular}

Table 1. Chemical bonds associated with biological processes and their approximate energies [28].

Anisotropy energy calculation was approximated to singledomain magnet anisotropy energy, with uniaxial anisotropy:

$$
E_{a n i}=K V \sin ^{2} \theta
$$

where $\mathrm{K}$ is the anisotropy constant, $\mathrm{V}$ is the particle volume and $\theta$ is the angle between the easy axis and the particle's magnetization.
The anisotropy constant $\mathrm{K}$ for magnetite nanoparticles consists of two values: from the volume of the inside core and from the surface [29]:

$$
K=K_{b}+\frac{S}{V} K_{S}
$$

where $K_{b}$ is the bulk anisotropy energy per unit volume, $S$ and $\mathrm{V}$ are the surface and the volume of nanoparticles, $\mathrm{K}_{\mathrm{S}}$ is the surface density of anisotropy energy. The values of anisotropy constant $\mathrm{K}$ for magnetite nanoparticles with different surfactants are in the range $3.1-4.3 \times 10^{4} \mathrm{Jm}^{-3}$, while for bulk magnetite are in the range $1.1-1.35 \times 10^{4}$ $\mathrm{Jm}^{-3}[29]$.

\section{RESULTS}

Magnetization of magnetite nanoparticles, for different sizes $(\mathrm{a} 1=4 \mathrm{~nm}, \mathrm{a} 2=11.5 \mathrm{~nm}, \mathrm{a} 3=47.7 \mathrm{~nm}, \mathrm{a} 4=150 \mathrm{~nm})$, and for strong magnetic field is shown in Fig.1a-d.
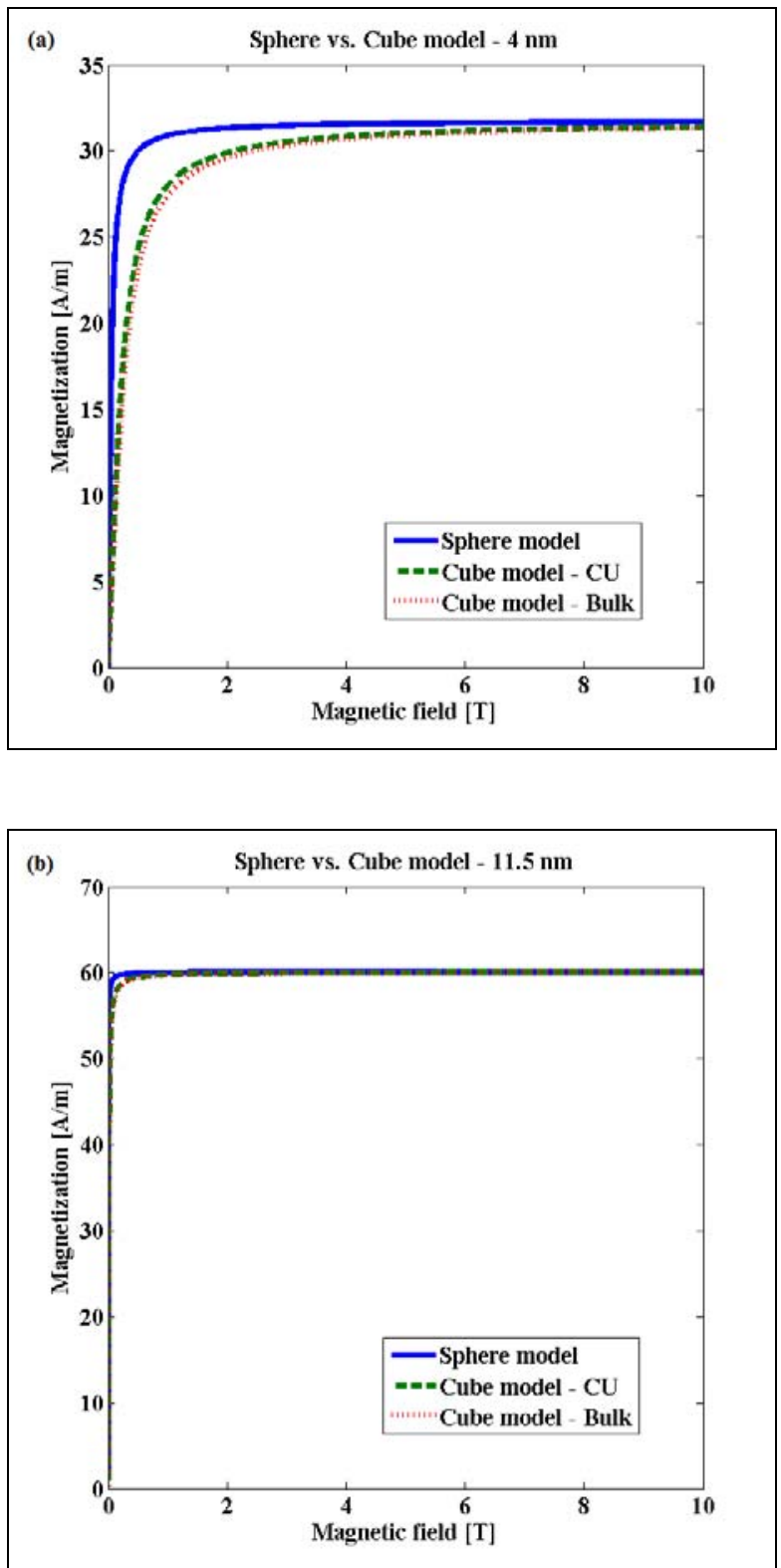

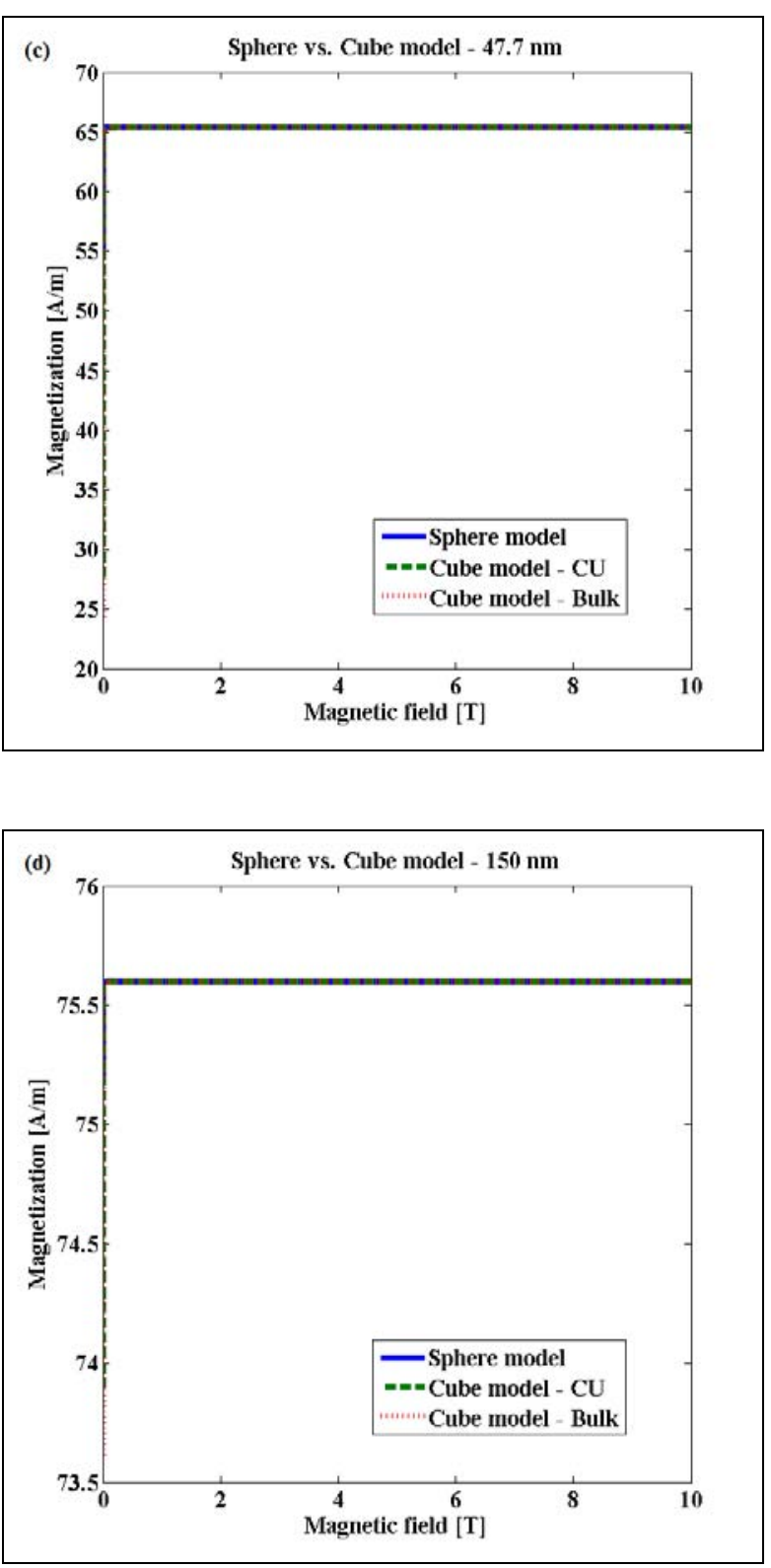

Fig.1. Comparison of magnetite nanoparticle magnetization values for generally used 'Sphere' model and our proposed 'Cube' model with cell unit (CU) and bulk approaches, in magnetite nanoparticles with different sizes: (a) $4 \mathrm{~nm}$, (b) $11.5 \mathrm{~nm}$, (c) 47.7 $\mathrm{nm}$, and (d) $150 \mathrm{~nm}$.

It is obvious that the difference between 'Sphere' model and 'Cube' model is insignificant for the strong magnetic fields and bigger particles. However, using an inappropriate model can lead to incorrect results for smaller particles and weak magnetic fields. The 'CU' approach and 'Bulk' approach of the 'Cube' model are almost identical, although they are principally different in the way of calculation. This was also a verification of appropriateness of the 'Cube' model approach. Therefore, in next simulations we used only one of them, the Cube - CU approach.

In Fig.2 is shown 'Sphere' vs. 'Cube' model energy comparison for four types of main MR magnetic fields.

Translation energy of magnetite nanoparticles in strong external magnetic field is shown in Fig.3. Dot lines represent energy level of biological bonds. We can see that for particles above $50 \mathrm{~nm}$, the translation energy, associated with magnetic gradients, exceed the value of the most powerful biological bond - covalent III.

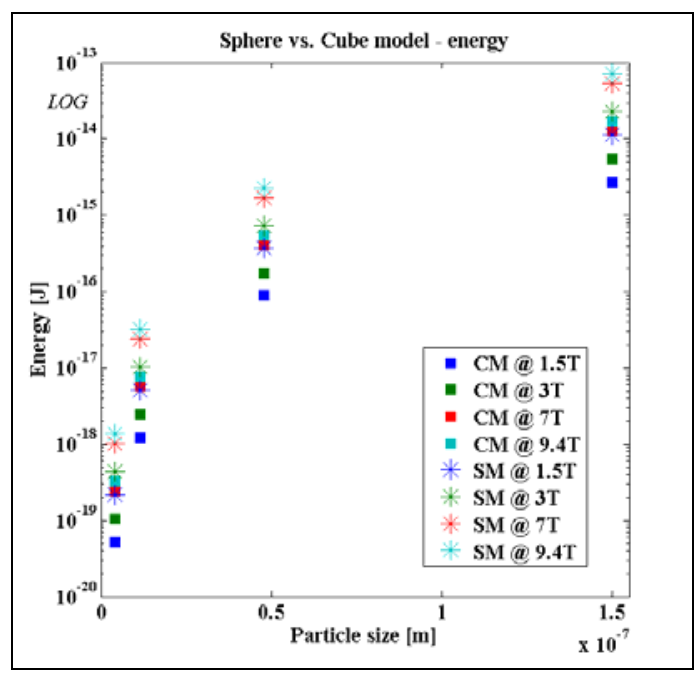

Fig.2. Comparison of energy (magnetic rotational work) for 'Sphere' model (SM) and 'Cube' model (CM) particles in magnetic resonance main fields: $1.5,3,7$ and 9,4T.

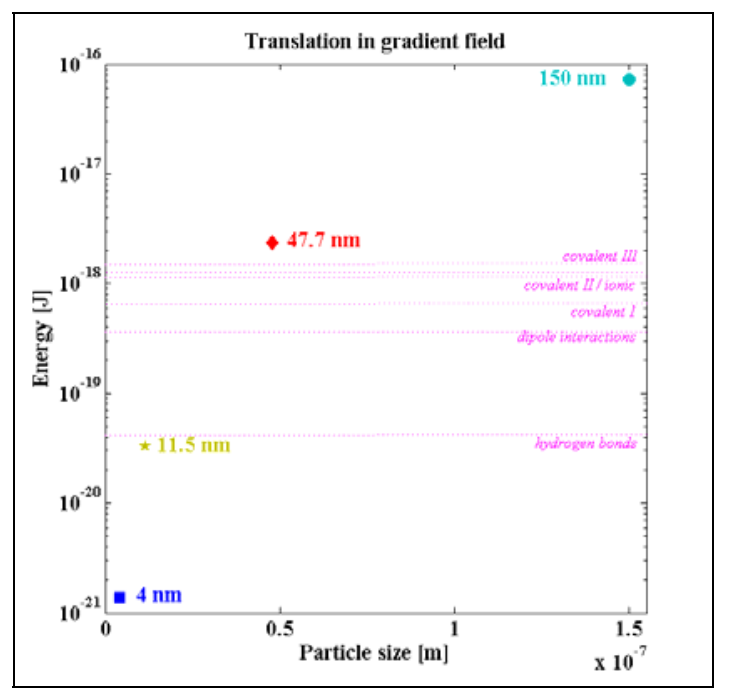

Fig.3. Translational energy of magnetite nanoparticles in magnetic gradient field $40 \mathrm{mT} / \mathrm{m}$, with biological bond energy lines.

Energies associated with interaction of magnetite nanoparticles with external static magnetic fields (magnetic rotational work) are shown in Fig.4. For all particles there exists critical value in magnetic field, when interaction energy exceeds anisotropy energy of particle (Table 2).

\begin{tabular}{|c|c|}
\hline Particle size [nm] & $\mathbf{B}_{\text {critical }}[\mathbf{m T}]$ \\
\hline 4 & 24.7861 \\
\hline 11.5 & 24.7838 \\
\hline 47.7 & 24.7839 \\
\hline 150 & 24.8489 \\
\hline
\end{tabular}

Table 2. Critical magnetic field values, in which magnetic rotational energy exceeds the anisotropy energy of particle. 


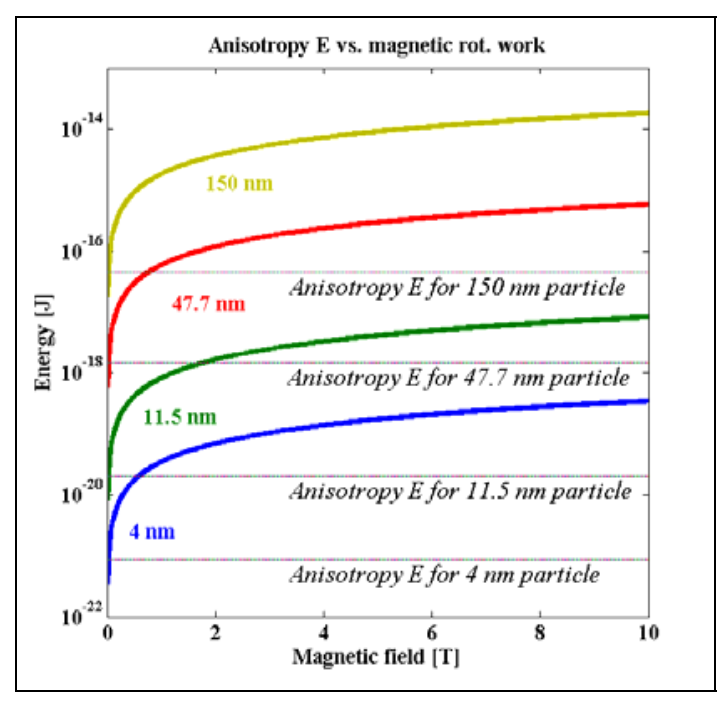

Fig.4. Magnetic rotational energy of magnetite nanoparticles in external static magnetic field, with anisotropy energy lines (calculated for $\theta=\pi / 2$ ).

Energies associated with particle interaction with static magnetic field of MRI scanners, with comparison to anisotropy energy and interaction with geomagnetic field, are shown in Fig.5.

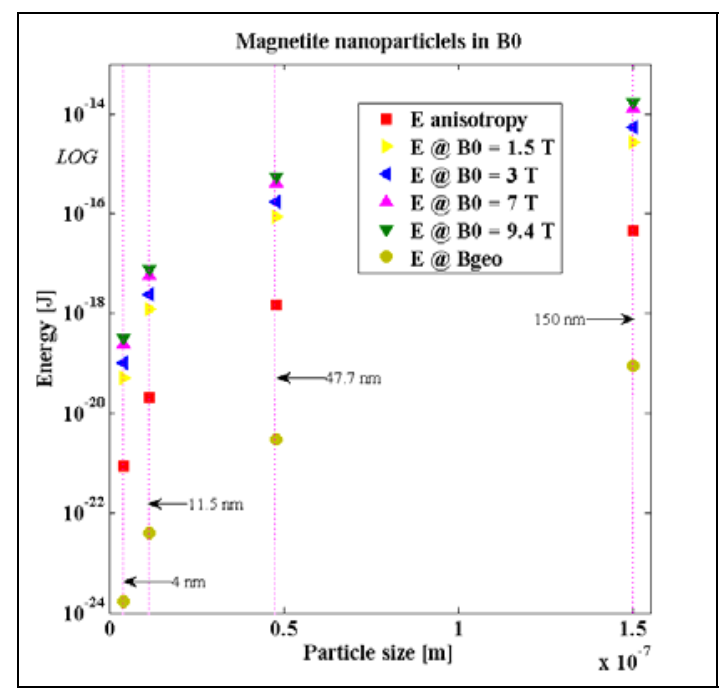

Fig.5. Magnetic rotational energy of magnetite nanoparticles in static magnetic field of MRI scanners, with anisotropy energy and geomagnetic field interaction energy (calculated for $\theta=\pi / 2$ ).

\section{DisCUSSION}

Magnetization curves in Fig.1a-d show difference between the generally used Sphere model and our proposed Cube model. However, 'cell unit' approach and 'bulk' approach in the Cube model are almost identical, although the method of calculation is completely different. Therefore, we believe that the Cube model represents a more realistic approach in magnetite nanoparticle behaviour simulations, and apart from evaluation of electromagnetic hazard, it can be helpful in body-iron quantification, determination of contrast agent efficiency, or other issues related to iron oxide nanoparticles. Energies associated with magnetic rotation work for Sphere model and Cube model particles are shown in Fig.2. For particles with sizes 4, 11.5 and $47.7 \mathrm{~nm}$, the differences between energies are not very distinct, but for particles bigger than $100 \mathrm{~nm}(150 \mathrm{~nm})$ the difference is quite extensive. And the use of incorrect model can lead to incorrect conclusions. In the following simulations, to determine magnetic moment of magnetite nanoparticles, only the Cube model was used, with 'cell unit' approach.

As mentioned in part ' 2 . Subject and methods', the magnetite nanoparticles in human tissue face, during MR examination, three principal fields: (i) static magnetic field $\mathrm{B}_{0}$, which causes alignment (rotation) of particles, (ii) gradient field, which induces translation motion of particles, and (iii) radiofrequency $B_{1}$ field, which causes primarily thermal effects. We have focused on the first two interactions. In Fig. 3 we show translation energy of particles in magnetic gradient field $\mathrm{B}_{\mathrm{grad}}=40 \mathrm{mT} / \mathrm{m}$, in comparison with energy levels of biochemical bonds. As we can see, energy of particles above $50 \mathrm{~nm}$ exceed all the biochemical bond energies $\left(\approx 10^{-19} \mathrm{~J}\right): 4 \mathrm{~nm} \approx 10^{-22} \mathrm{~J}, 11.5 \mathrm{~nm} \approx 10^{-20} \mathrm{~J}$, $47.7 \mathrm{~nm} \approx 10^{-18} \mathrm{~J}, 150 \mathrm{~nm} \approx 10^{-17} \mathrm{~J}$. The interaction of particles with main magnetic field $\mathrm{B}_{0}$ is much more powerful, and does not vary too much with particle size. Energies associated with such interaction are in the order of $10^{-14} \mathrm{~J}$ for fields up to $7 \mathrm{~T}$ and $10^{-13} \mathrm{~J}$ for field $9.4 \mathrm{~T}$. Energies of such interaction with comparison to anisotropy energy and interaction energy with geomagnetic field are shown in Fig.5. Very interesting is the fact, that interaction energy of particles with geomagnetic field is in the same order as biochemical bond energies, $\approx 10^{-19} \mathrm{~J}$. In Fig.4 energy curve of particles in external magnetic field up to 10 $\mathrm{T}$ is shown. As is obvious, there is critical point for each particle, in which magnetic interaction energy exceeds the anisotropy energy of particle. The values are shown in Table 2, and from $11.5 \mathrm{~nm}$ they increase with particle size increase.

Biogenic magnetite in human tissue represents biologically produced ferrimagnetic nanoparticles. We do not know its function, but if we accept, that in animals it plays a crucial role in geomagnetic field navigation, we also have to accept interaction with neurosensory system through biochemical bonds. As we have shown, biogenic magnetite interaction with fields inside MR devices exceeds biochemical bond energies, both for interaction with static and gradient magnetic field. Disruption of coupling with sensory cell membranes releases particles to the surrounding environment. Interaction energies of free ferrimagnetic particles, with extreme fields of MR devices, can be a serious hazard issue, especially in sensitive tissue like the human brain is. The bigger the particle is, the bigger is also the risk hazard. Schultheiss-Grassi et al. reported particles with diameter up to $500 \mathrm{~nm}$ [10]. Moreover, Vaughan has shown, that free magnetite nanoparticles can in specific pulse magnetic fields create the hydrophilic pores in cell membrane [17]. Hydrophilic pores are devastating for cell integrity and can cause cell decease. Specific conditions mean that pulse magnitude must exceed $\mathrm{B} \approx 6 \times 10^{-3} \mathrm{~T}$ to $7 \times 10^{-2} \mathrm{~T}$, and the pulse duration is in the range $10^{-5}$ to $10^{-1}$ 
seconds. In standard MR devices you easily fulfil these 'specific' conditions.

To summarize, biogenic magnetite nanoparticles inside human brain tissue are, during MR examination, affected with three principal fields:

(i) Static magnetic field $\mathrm{B}_{0}$, which causes alignment (rotation) of particles with the field direction, and energy associated with such interaction is in order 5 times higher than biochemical bond energy

(ii) Gradient magnetic field $B_{\text {grad }}$ (which encodes the spatial position of the signal), which causes translation movement of particles, and interaction energy exceeds the biochemical bond energy in particles bigger than $50 \mathrm{~nm}$ (for $1.5 \mathrm{~T}$ field). Moreover, Cavopol has shown that the principal determinant of action potential blockade in sensory neurons is the field gradient, not intensity [30].

(iii) Radiofrequency pulses ( $\mathrm{B}_{1}$ field), cause thermal effect (heating) in tissue, which can be multiplied by particle relaxation processes (hyperthermia) [31].

And free ferrimagnetic nanoparticles inside human brain tissue are, during MR examination, a potential risk factor in magnetic resonance hazard questions.

\section{CONCLUSIONS}

The biological processes are time and frequency dependent and are generally nonlinear in their response to applied fields [26]. Together with subject-dependent physiological response, it is difficult to predict any threshold parameters. Without precise knowledge of biogenic magnetite physiological function and interaction with surrounding environment, it is more or less hypothetical to describe precise mechanisms of hazardous interaction. However, our preliminary simulations indicate potential health hazard impact of such interaction. Moreover, the results show one very interesting fact. The interaction energy of magnetite nanoparticles with geomagnetic field is in the same order as the energy of biochemical bonds in living systems $\left(\approx 10^{-19} \mathrm{~J}\right)$. This supports the theory of geomagnetic field navigation and magnetoreception of animals, with the help of biogenic magnetite nanoparticles [32].

To understand the precise mechanism of interaction in humans, we need to clarify two basic things. First, what is the physiological function of such particles and what is their spatial arrangement in tissue. Second, what is the biological response of organism to applied fields? We showed that biogenic magnetosomes can interact with geomagnetic field and in MR fields they can cause mechanical damage. On the other hand, what will be the reaction of organism on the molecular, cellular or higher level? Weaver described the phenomenon, when we can expect the effect of nonionizing EM fields to biological system, as follows: "a physical field can create a biological effect only through altering one or more chemical processes within the biological system" [33]

\section{PHYSICAL FIELD $\rightarrow$ CHEMICAL PROCESS $\rightarrow$ BIOLOGICAL SYSTEM}

Can such kind of interaction influence the process of biochemical reactions? Kirschvink in 1994 proposed magnetic steering of chemical reactions by magnetite nanoparticles [34]. Can such a modification be responsible for pathological changes (e.g., creation of plaques in Alzheimer disease because of increasing electromagnetic smog)? We cannot answer this question at present time. However, we can propose a new approach. The external field - as a nonmaterial energy, primarily interacts with magnetic field of particles and magnetic field of bioelectric currents (ion flow in neurons):

\section{PHYSICAL FIELD $\rightarrow$ BIOLOGICAL RESPOND $\rightarrow$ (CHEMICAL PROCESS CHANGE)}

Result of such interaction, if extremely strong, can be visible on the physical level - as a change in chemical reaction process, with new chemical products. This affects mainly organisms on molecular level, with consequences on physiology of cell, tissue, and organ. However, weakness interaction is not visible in matter, but can influence the human as a unit - rapid change of mood or concentration, unpleasant feelings, disorientation, rapid heartbeat, head ache, loss of appetite, tiredness, stress, etc. These are "normal" demonstrations of civilized people, who live in towns with enormous increase of artificial (electro-) magnetic fields [35]. The situation in MR devices is much more extreme, so it can be more "detectable" and can bring new views to the problem of (electro-) magnetic field interaction with living systems [36].

\section{ACKNOWLEDGMENT}

This work was supported by the Slovak Scientific Grant Agency VEGA 2/0090/11 and State program SPVV no. 2003 SP200280203.

\section{REFERENCES}

[1] Banerjee, S.K., Moskowitz, B.M. (1985). Ferrimagnetic properties of magnetite. In Kirschvink, J.L. (ed.) Magnetite Biomineralization and Magnetoreception in Organisms: A New Magnetism. Plenum Publishing Corporation, 17-41.

[2] Lowenstam, H.A. (1962). Magnetite in denticle capping in recent chitons (polyplacophora). Bulletin Geological Society of America, 73, 435-438.

[3] Safarik, I., Safarikova, M. (2002). Magnetic nanoparticles and bioscience. Monatshefte fur Chemie, 133, 737-759.

[4] Kirschvink, J.L., Hagadorn, J.W. (2000). A grand unified theory of biomineralization. In Bauerlein, E. (ed.) The Biomineralization of Nano- and MicroStructures. Weinheim, Germany: Wiley, 139-150.

[5] Thomas-Keprta, K. et al. (2000). Elongated prismatic magnetite crystals in ALH84001 carbonate globules: Potential Martian magnetofossils. Geochimica et Cosmochimica Acta, 64, 4049-4081.

[6] Walker, M.M. (2008). A model for encoding of magnetic field intensity by magnetite-based magnetoreceptor cells. Journal of Theoretical Biology, 250, 85-91.

[7] Kirschvink, J.L., Kobayashi-Kirschvink, A., Woodford, B.J. (1992). Magnetite biomineralization in 
the human brain. Proceedings National Academy of Sciences USA, 89, 7683-7687.

[8] Dunn, J.R. et al. (1995). Magnetic material in human hippocampus. Brain Research Bulletin, 36, 149-153.

[9] Dobson, J., Grassi, P. (1996). Magnetic properties of human hippocampal tissue: Evaluation of artefacts and contamination sources. Brain Research Bulletin, 39, 255-259.

[10] Schultheiss-Grassi, P., Weissiken, R., Dobson, J. (1999). TEM investigations of biogenic magnetite extracted from human hippocampus. Biochimica and Biophysica Acta, 1426, 212-216.

[11] Schultheiss-Grassi, P.P., Heller, F., Dobson, J. (1997). Analysis of magnetic material in the human heart, spleen and liver. Biometals, 10, 351-355.

[12] Hautot, D., Pankhurst, Q.A., Khan, N., Dobson, J. (2003). Preliminary evaluation of Nanoscale biogenic magnetite in AD brain tissue. Proceedings of the Royal Society London B (Biology Letters), 270, S62-S64.

[13] Collingwood, J.F. et al. (2005). In-situ characterization and mapping of iron compounds in Alzheimer's tissue. Journal of Alzheimer's Disease, 7, 267-272.

[14] Pankhurst, Q.A., Hautot, D., Khan, N., Dobson, J. (2008). Increased levels of magnetic iron compounds in Alzheimer's disease. Journal of Alzheimer's Disease, 13, 49-52.

[15] Dobson, J. (2002). Investigation of age-related variations in biogenic magnetite levels in the human hippocampus. Experimental Brain Research, 144, 122-126.

[16] Bartzokis, G., Sultzer, D., Cummings, J., Holt, L.E., Hance, D.B., Henderson, V.W., Mintz, J. (2000). In vivo evaluation of brain iron in Alzheimer disease using MRI. Archives of General Psychiatry, 57, 47-53.

[17] Vaughan, T.E., Weaver, J.C. (1996). Energetic constraints on the creation of cell membrane pore by magnetic particles. Biophysical Journal, 71, 616-622.

[18] Strbak, O., Gogola, D., Frollo, I. (2011). Cube model approach in simulating of magnetite nanoparticles behaviour in external magnetic fields. In MESUREMENT 2011: $8^{\text {th }}$ International Conference on Measurement, April 27-30, 2011. Bratislava: Institute of Measurement Science SAS.

[19] Huang, D.J. et al. (2004). Spin and orbital magnetic moments of $\mathrm{Fe}_{3} \mathrm{O}_{4}$. Physical Review Letters, 93 (7), 077204.

[20] Goya, G., Berquo, T., Fonseca, F., Morales, M. (2003). Static and dynamic magnetic properties of spherical magnetite nanoparticles. Journal of Applied Physics, 94 (5), 3520-3528.

[21] Chen, C.H., Abate, A.R., Lee, D., Terentjev, E.M., Weitz, D.A. (2009). Microfluidic Assembley of magnetic hydrogel particles with uniformly anisotropic structure. Advanced Materials, 21, 3201-3204.

[22] Shellock, F.G. (2001). Metallic neurosurgical implants: evaluation of magnetic field interactions, heating and artifacts at 1.5-Tesla. Journal of Magnetic Resonance Imaging, 14, 295-299.

[23] New, P.F.J., Rosen, B.R., Brady, T.J. et al. (1983). Potential hazards and artifacts of ferromagnetic and non-ferromagnetic surgical and dental materials and devices in nuclear magnetic resonance imaging. Radiology, 147, 139-148.

[24] Kalambur, V.S., Han, B., Hammer, B.E., Shield, T.W., Bischof, J.C. (2005). In vitro characterization of movement, heating and visualization of magnetic nanoparticles for biomedical applications. Nanotechnology, 16, 1221-1233.

[25] Naeeni, H.A., Haghpanahi, M. (2009). Viscoelastic modeling of brain MRE using FE method. Engineering and Technology, 54, 726-729.

[26] Glover, P.M. (2009). Interaction of MRI field gradients with the human body. Physics in Medicine and Biology, 54, R99-R115.

[27] Triventi, M., Mattei, E., Calcagnini, G., Censi, F., Bartolini, P., Kainz, W., Bassen, H. (2007). Magnetic resonance induced heating of implantable leads. Annali dell'Istituto Superiore di Sanita, 43 (3), 229-240.

[28] Wolke, R.L. (2009). Bond energy. Retrieved from http://science.jrank.org/pages/984/Bond-Energy.html.

[29] Turcu, R., Pana, O., Nan, A., Craciunescu, I., Chauver, O., Payen, C. (2008). Polypyrrole coated magnetite nanoparticles from water based nanofluids. Journal of Physics D: Applied Physics, 41, 245002.

[30] Cavopol, A.V., Wamil, A.W., Holcomb, R.R., McLean, M.J. (1995). Measurement and analysis of static magnetic fields that block action potentials in cultured neurons. Bioelectromagnetics, 16, 197-206.

[31] Timko, M. et al. (2009). Magnetite properties and heating effects in bacterial magnetite nanoparticles. Journal of Magnetism and Magnetic Materials, 321, 1521-1524.

[32] Kirschvink, J.L., Walker, M.M., Diebel, C.E. (2001). Magnetite-based magnetoreception. Current Opinion in Neurobiology, 11, 462-467.

[33] Weaver, J.C. (2002). Understanding conditions for which biological effects of nonionizing electromagnetic field can be expected. Bioelectrochemistry, 56, 207-209.

[34] Kirschvink, J.L. (1994). Rock magnetism linked to human brain magnetite. Transaction American Geophysical Union, 75, 178-179.

[35] Warnke, U. (2007) Birds, Bees and Mankind: Destroying Nature by 'Electrosmog'. A Brochure Series by the Competence Initiative for the Protection of Humanity, Enviroment and Democracy.

[36] Starcuk, Z., Bartusek, K., et al (2006). Evaluation of MRI artifacts caused by metallic dental implants and classification of the dental material in use. Measurement Science Review 6(2), 24-27. 